\title{
Optimization of Energy Consumption in Wireless Sensor Networks based on Nature-Inspired Algorithms
}

\author{
Nizar Hadi Abbas, Ph.D \\ Electrical Engineering \\ Department \\ College of Engineering, \\ University of Baghdad, Iraq.
}

\author{
Tarik Zeyad Ismaeel,Ph.D \\ Electrical Engineering \\ Department \\ College of Engineering, \\ University of Baghdad, Iraq.
}

\author{
Rassim Nooraldin Ibrahim \\ Electrical Engineering \\ Department \\ College of Engineering, \\ University of Baghdad, Iraq.
}

\begin{abstract}
Wireless Sensor Networks (WSNs) consists of a huge number of tiny, low-priced, and battery-powered devices with limited on board sensing, processing and communication capabilities. The batteries of sensor nodes of WSNs are usually with limited capacity; hence it is essential to conserve battery energy to prolonging the WSNs lifetime. Therefore, this paper deals with the matter of energy consumption minimization to maximize the overall network lifespan. In this research, a mathematical model for the lifetime of WSN is formulated based on several parameters to find out the optimal solution of the energy problem in the field of wireless sensor networks using the Modified Particle Swarm Optimization (MPSO) and Ant Colony Optimization (ACO) algorithms. The proposed system has been implemented using MATLAB 7.6.0 (R2008a) software environment. The computer simulation results show that the presented approach for power consumption minimization is faster than the previous works by 10 times, and the network lifetime is increased by at least 8 times. Furthermore, the conducted simulation indicates that the MPSO algorithm offers superior results in terms of accuracy (99.36\%) in comparison with ACO algorithm $(97.92 \%)$. In this regards, MPSO algorithm acts with much better efficiency as computational time minimizes, simple, has stable convergence characteristics, and designed with adaptable inertial weight and acceleration factors than ACO algorithm.
\end{abstract}

\section{Keywords}

Wireless Sensor Networks, Energy Efficiency, Network Lifetime, WSN Modeling, Nature-Inspired Algorithms, PSO Algorithm, ACO Algorithm.

\section{INTRODUCTION}

Wireless sensor networks are widely used in the common fields of the current science life, so they had a lot of interest concerning monitoring systems in science researches, military, agricultural and astronomy ... etc. The matter of power is the most crucial issue in that field of work so that, many studies discussed and treated the problem of the power consumption in different approaches using different optimization algorithms [1].

L. Bai, et al. [2], designed a protocol for cooperative WSNs with energy balance consideration. Since the design of WSNs is highly dependent on application scenarios, the effects of system parameters are thoroughly analyzed and a unified criterion is established to choose the proper cooperative scheme. Moreover, energy balance is achieved by adjusting the size of clusters. They classified energy consumption into two groups: inter-cluster energy consumption and intra-cluster energy consumption. Sensor nodes consuming higher energy in inter-cluster data communication form smaller clusters. Thus energy consumption is balanced between intra- and inter-cluster data communication.

F. Bagci, et al. [3], proposed energy efficient medium access control (MAC) protocol for wireless sensor networks, called Energy Saving Token Ring Protocol (ESTR). ESTR bases on the well-known token ring protocol. The sensor nodes are connected in rings where only the node that holds the token is active and able to communicate. ESTR establishes sleep times for sensor nodes that don't need to send or receive messages. Thus, nodes save energy spending with less time in active mode.

A Power Saving Scheme (PSS) multi-hop sensor network using partition clusters is proposed by S. Anandamurugan and C. Venkatesh [4]. It avoids collision and idle listening time of sensor nodes for better overall network life time. The basic sensor nodes are simple and have limited power supplies, whereas the cluster head nodes are much more powerful and have many more power supplies, which organize sensors around them into clusters. Such two-layered heterogeneous sensor networks have better scalability and lower overall cost than homogeneous sensor networks. It is proposed that using polling to collect data from sensors to the cluster head since polling can prolong network life by avoiding collisions and reducing the idle listening time of sensors.

C. Srimathi, et al. [5], proposed EAR (Energy Aware Routing), which is a novel routing protocol for under water sensor networks. It provides real-time, reliable delivery of a packet, while considering energy awareness. In EARQ, a node estimates the energy cost, delay and reliability of a path to the sink node, based only on information from neighboring nodes. Then, it calculates the probability of selecting a path, using the estimates. When packet forwarding is required, it randomly selects the next node. A path with lower energy cost is likely to be selected, because the probability is inversely proportional to the energy cost to the sink node. To achieve real-time delivery, only paths that may deliver a packet in time are selected.

An energy efficient scheduling algorithm for clustered wireless sensor networks is presented by J. K. Murthy, et al. [6]. The main objective is to provide optimized Time Division Multiple Access (TDMA) schedules that can acquire high 
power efficiency, reduced interference, reduced conflict and reduced end-to-end delay over a wide network. To obtain this objective, a joint optimal design of network, medium access control (MAC) and physical layers is considered to reduce the overall energy consumption. The slot reuse concept is applied to derive the TDMA schedule to minimize the frame length.

In this paper we proposed a new model that combines both a power consumption issue with a nature inspired algorithm. In this model, we take under consideration all previous WSNs models' parameters like adjustable transmission ranges and corona usage, adjustable sensing ranges, hexagonal deployment mode per each mini-cluster, and usage of both Omni \& directional antennas in the same cluster to transmit the information directly from the particular node to the sink node. The optimization process of the WSN model has been done by utilizing the PSO \& ACO algorithms. Subsequently, the optimum values collected form PSO algorithm is compared with those extracted from ACO to validate the achieved results; these results may help network's creators to choose the perfect parameters for their network nodes in order to maximize the lifetime of WSNs to overcome the power consumption problem.

The remainder of this paper is organized as follows: section 2 explains the theoretical basics of the WSNs and natureinspired algorithms; section 3 describes the mathematical model of WSN; section 4 presents the application of MPSO and ACO algorithm on WSN model to maximize the network lifetime and the simulation results are provided in section 5 . Finally, section 6 outlines the conclusions of the research.

\section{THEORETICAL BASICS}

\subsection{Wireless Sensor Networks}

A Wireless Sensor Network can be generally described as a group of sensor nodes arranged and organized into a cooperative manner network that can sense and control the environment enabling interaction between persons or embedded computers and the surrounding environment events. Generally, a WSN is a compound of hundreds of multifunctional sensing nodes densely deployed in some geographical area and one or few base stations or sink nodes connect a sensor network to the users via the Internet or other transmission media networks.

Basically, each sensor node consists of a CPU (microcontrollers or microprocessors) for processing the data, memory for storage, an RF transceiver with a single Omni or directional antenna and a power source like batteries or sometimes solar cells. Typically, the task for such networks is to monitor temperature, light, pressure, vibration, humidity, sound, radiation ...etc. Each of these sensor nodes acquires the data from the field, process it and route it to the sink node by a single or multi-hopping way [7]. Wireless sensor networks' technology was growing in the recent years in both industry and academia to investigate different practical phenomena based on the emerging technologies.

\subsection{Nature-Inspired Algorithms}

Nature-inspired algorithms are the main part of advanced evolutionary optimization algorithms, which have drawn notable interest in the last decade. Many types of natureinspired algorithms such as Particle Swarm Optimization (PSO), Bee Colony Optimization (BCO) and Ant Colony Optimization (ACO) have been introduced. They have rapidly progressed in recent few years and have presented and applied on many successful applications to solve real-world optimization problems.
On the other hand, conventional optimization algorithms such as steepest descent (SD), Newton and successive quadratic programming (SQP) have been applied in a wide variety of optimization problems. However, in some cases they have been confronted with same problems such as an improper initial guess. Additionally, they cannot be used in discrete systems [8].

The PSO algorithm has been used to solve a lot of optimization problems, because of its powerful capabilities, ease of implementation and fast convergence speed [9]. The ACO algorithm has been applied to a broad range of hard combinatorial problems, and these problems are defined in terms of components and states. In this regard, ACO can generate an optimum solution path in case of dynamically adding new components to a state [8].

\subsubsection{PSO Algorithm}

Particle swarm optimization algorithm is invented by $\mathrm{J}$. Kennedy and R. Eberhart [9], based on the social behavior of collection of animal such as birds flocking and fish schooling. Each solution in PSO algorithm is considered as a 'bird' in the flock and is referred to as a 'particle'. A particle is analogous to a chromosome (population member) in GA's (Genetic Algorithms). As compared to GA's, the evolutionary process in the PSO does not create new birds from parent ones. Rather, the birds in the population only evolve their social behavior and accordingly, their movement towards a destination [10]. In PSO algorithm, each particle in the swarm, remembers the best solution found by itself and by the whole swarm along the search trajectory. The particles move through the search space and exchange information with other particles according to the following equations [10]:

$$
\begin{aligned}
& V_{i d}(\text { next })=w * V_{i d}(\text { current })+c_{1} * r_{1} *\left(P_{\text {best }}-X_{i d}\right) \\
& +c_{2} * r_{2} *\left(g_{\text {best }}-X_{\text {id }}\right) \\
& X_{i d}(\text { next })=X_{i d}(\text { current })+V_{i d}(\text { next })
\end{aligned}
$$

Where $X_{i d}$ represents the current position of the particle, $\mathrm{P}_{\text {best }}$ is the best remembered individual particle position, $\mathrm{g}_{\text {best }}$ denote the global best position of the whole particles entire the swarm. $c_{1}$ and $c_{2}$ are cognitive and social parameters. $r_{1}$ and $r_{2}$ are random numbers between 0 and 1 and $w$ is inertial weight which is used to balance the global and local search abilities. A large inertia weight facilitates global search while a small inertia weight facilitates local search [9].

\subsubsection{MPSO Algorithm}

The modified particle swarm optimization algorithm is developed based on the inertial weight $w$ and the acceleration $c_{1}$ and $c_{2}$ which are not fixed values and updated during the PSO operation by some equation or relationship, then the PSO search process will modified accordingly $[10,11]$.

In this work, MPSO is considered and the update equations for the inertia weight and acceleration factors are as below:

$$
\begin{aligned}
& w=w_{\text {max }}-\text { alpha } 1 * r 1 *\left(w_{\text {min }}\right)^{\text {beta } 1} \\
& c_{1 \text { next }}=c_{1 \text { cuurent }}-\text { alpha } 2 * r 2 *\left(c_{1 \text { cuurent }}\right)^{\text {beta } 2} \\
& c_{2 \text { next }}=c_{2 \text { cuurent }}-\text { alpha } 3 * r 3 *\left(c_{2 \text { cuurent }}\right)^{\text {beta } 3}
\end{aligned}
$$


Where alpha1, alpha2, alpha3, beta1, beta 2 \& beta 3 are factors determined by us according to the problem, and $r 1, r 2$ $\& r 3$ are random values between $0 \& 1, W_{\max }$ is the maximum value of the inertial weight and equal to 0.9 and the $W_{\min }$ is the minimum limit value for the inertia weight and equal to 0.4 , $c_{1 \text { cuurent }} \& c_{2 \text { cuurent }}$ are acceleration factors and their values usually between $1.5 \& 2$ [11].

\subsubsection{ACO Algorithm}

Ant colony optimization algorithm is a proposed metaheuristic approach for solving hard combinatorial optimization problems. ACO was inspired from the principle of pheromone trail lying and following behavior of real ants, which use pheromones as a communication source and medium. Analytically the biological example described the ACO algorithm behavior is related to the indirect contact of a colony of simple agents, called (artificial) ants, mediated by (artificial) pheromone trails. The pheromone trails in ACO same as distributed numerical information, which is used by the ants to probabilistically construct solutions for the given problem. Furthermore, this chemical substance is used by the ants to adapt the search process during the algorithm's execution to reflect their search experience [12].

The ACO algorithm has been widely applied to solving various combinatorial optimization problems such as Traveling Salesman Problem (TSP), Job-shop Scheduling Problem (JSP), Vehicle Routing Problem (VRP), Quadratic Assignment Problem (QAP), etc. [13]. Although ACO has a powerful capacity to find out solutions to combinational optimization problems, it has the problems of stagnation and premature convergence as well as the convergence speed of ACO is very slow. Those problems will appear when the problem size increases. Therefore, several extensions and improvements' versions of the original ACO algorithm were introduced over the years [13]. The ants move through the search space and exchange information with others and evaporate the pheromone amount to guide other ants according to the following equations [12]:

$P_{i j}=\frac{\tau_{i j}^{(l)}}{\sum_{j=1}^{n} \tau_{i j}^{(l)}} ; i=1,2, \ldots ., ; j=1,2,3 \ldots \ldots, n$;

$\tau_{i j}^{(l)}=\tau_{i j}^{(o l d)}+\sum_{k} \Delta \tau_{i j}^{(k)}$

$\tau_{i j}^{(o l d)}=(1-\rho) * \tau_{i j}^{(l-1)}$

$\sum_{k} \Delta \tau_{i j}^{(k)}=\frac{\delta * F_{\text {best }}}{F_{\text {worst }}}$

Where $l$ is representing the iteration number, $\tau_{i j}^{(o l d)}$ denotes the pheromone amount of the previous iteration left after evaporation, $\sum_{k} \Delta \tau_{i j}^{(k)}$ is the pheromone deposited by the best ant $k$ and the summation extends over all the best ants $k$ (if multiple ants take the best path), $\rho$ the pheromone decay factor and $\delta$ is the scaling parameter.

\section{Modeling of Wireless Sensor \\ Network}

In this research, a new WSN model is developed to enhance and prolong the network overall lifetime. This model assuming that there is a network working for some period of time depending upon the battery energy then this proposed system will find a way to increase the working lifetime of the same network by changing some parameters. So the basic issue is that the same number of nodes will be used, but may be the radiuses of transmission and sensing will be changed in addition to the scheme of the deployment. The first step of the proposal is the deployment of the nodes. Each group of nodes should be arranged in some way in a cluster; this cluster will conserve the energy consumption and provide enough coverage for its area.

In previous work [14], some proposed models were introduced, A-3 is arranging the nodes as a triangular shape and one small node at the middle to cover the gap as detailed in Figure 1a, B-3 is as a square shape and one small node at the middle to cover the gap as in Figure 1b. Consequently, we developed here a new model and calculate the energy for it and find out the differences between the proposed model and the aforesaid models. The proposed model is a hexagonal shape for six equal nodes as illustrated in the Figure 2. The sensing consumed energy is described in Eq. (10) for a multiple number of nodes arranged in hexagonal shape.

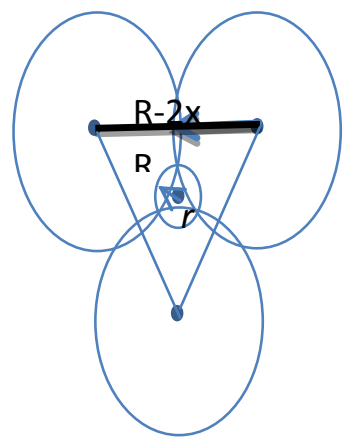

Figure 1a: A-3

Triangular model

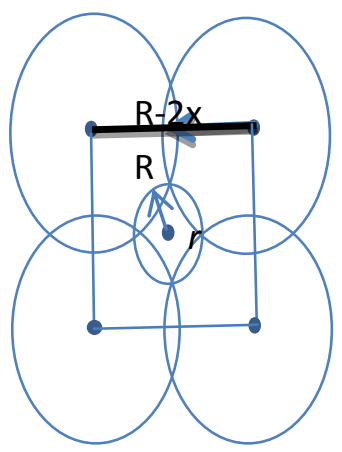

Figure 1b: B-3 Square model
Figure 1:The previous proposed shapes for clustering and coverage

$E_{\text {Sensing }_{\text {total }}}=1.11 * R^{2} * \mu_{2}$

In this work, a full-coverage model was performed and with good small consumed energy, which is better than B-3 and a bit more energy consumption than that in A-3 by a small ratio, but the design for a network will be easier and will include more nodes with more coverage area, also this model can be

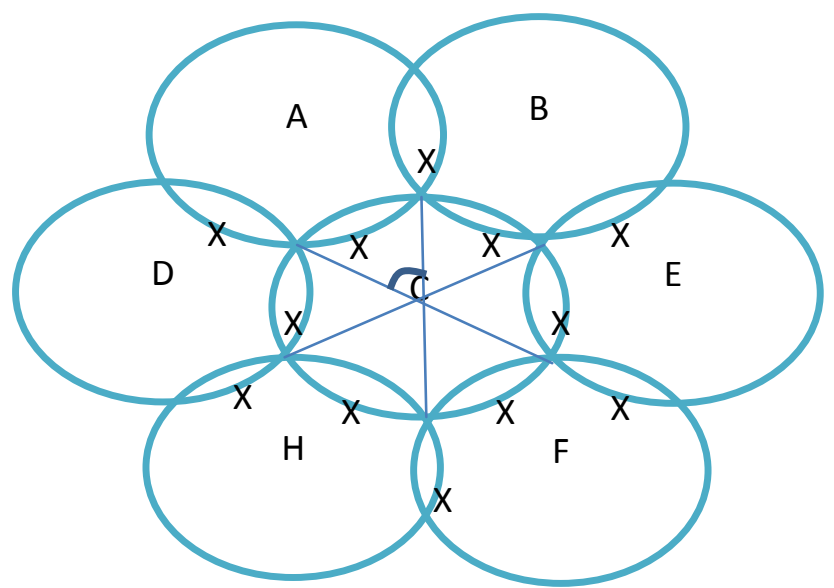

Figure 2: The hexagonal mode for seven nodes 
modified to get less than $100 \%$ coverage with less energy consumed per each group of neighbors and this case is valid when some tradeoff between coverage and energy consumption is occurred. Then the energy consumption $E_{c}$, is as below:

$E_{c}=E_{T X}+E_{\text {Link }}$

Where $E_{\text {Link }}$ is the consumed energy in the link between the nodes and sink node, and $E_{T X}$ is the energy of transmission the data from node to node (Hop-By-Hop transmission).

$E_{\text {Link }}=h * E_{T X}+(h-1) * E_{R X}$

$E_{T X}= \begin{cases}l * E_{F S} * d^{2} & , d<d \\ l * E_{T r} * d^{4} & , d>d\end{cases}$

Where $E_{R X}$ is the energy consumed when the node receives data from neighbor node, $\mathrm{d}$ is transmission radius, and

$E_{F s}=10 \mathrm{pJ} / \mathrm{bit} \cdot \mathrm{m}^{2}$

$E_{T r}=0.0013 \mathrm{pJ} / \mathrm{bit} \cdot \mathrm{m}^{4}$

$E_{R x}=l * E_{\text {elec }}$

Where $l$ is the message length in bits, and

$E_{\text {elec }}=50^{\mathrm{nJ}} / \mathrm{bit}$

Then,

$E_{C}=\underbrace{E_{T x}}_{A}+\underbrace{h * E_{T x}+(h-1) * E_{R x}}_{B}$

In [15], three models are introduced; the target of proposing these models was to get the optimum coverage for all the area through the sensing nodes. The three models are developed with different energy consumption limits, because the main interest was on the energy consumption concept in addition to the network coverage. However, the transmission energy was not taken into consideration in the proposed model. From their three models, it can be concluded that the energy consumed during sensing operation is as below:

$\mathrm{E}_{\mathrm{s}}=\frac{\mathrm{r}_{\text {sensing }}^{4}{ }^{*} \mu_{2}}{\text { The area of this cluster }}$

Therefore, in our proposal, we fill this gap, by regarding that each node can consume energy depending on its radius and covered area. Then,

$E_{\text {sensing }}=\frac{r_{\text {sensing }}^{4} * \mu_{2}}{\pi * r_{\text {sensing }}^{2}}=\frac{1}{\pi} * r_{\text {sensing }}^{2} * \mu_{2}$

The usage of different $\&$ adjustable transmission ranges and each range will be regarded as a corona around the sink node, as well as adaptable sensing ranges was proposed. Depending on the previous models and the below comments, we build our mathematical model:

1. It is assumed that each node should transmit its own information to the sink node directly and should not use the hop-by-hop mode, so the receiving operation energy in all nodes will be removed (there is no hop-by-hop) transmission. Then the part B from Eq. (15) will be deleted and then we can conclude from Eqs. (15) and (17) the following equation:

$E_{C}=\underbrace{\frac{1}{\pi} * r_{\text {sensing }}^{2} * \mu_{2}}_{\text {Sensing part }}+\underbrace{l * r_{\text {transmission }}^{4} * E_{T r}}_{\text {Transmission part }}$

2. It is also assumed that this proposal will regard the system of clustering in the deployment of nodes and in the relations between nodes and their sink.

Then, we can calculate the required number of clusters per the field area which is depended on the maximum radius of transmission $R_{t \_ \text {Max }}$ as follows:

$A_{C}=r_{\text {Transmission }}^{2} * \pi=$ Area per each cluster

$N_{C}=\frac{A}{A_{C}}=$ Number of clusters required

Each cluster should contain $\mathrm{m}$ coronas depending on the transmission range $\mathrm{m}$. So it is assumed that there is a space of choosing the sensing radius ranges randomly over its interval to find the number of nodes required per each cluster for each value of sensing radius:

$A_{s}=\pi * r_{\text {Sensing }}^{2}=$ sensing area per node

$N_{S}=\frac{A_{C}}{A_{S}}=$ no. of nodes required per each cluster

Also it is required to calculate the number of nodes required per each corona:

$C_{i_{i=1 \text { tom }}}=\frac{\left(\pi * r^{2}{ }_{t_{i}}\right)-\left(\pi * r^{2}{ }_{t_{i-1}}\right)}{A_{S}}$ for $i=1$ then $r=0$

Where,

$C 1=$ Number of nodes required per corona 1

$C 2=$ Number of nodes required per corona 2

$C 3=$ Number of nodes required per corona 3

!

$\mathrm{Cm}=$ Number of nodes required per corona $m$

For sensing issue, isotropic or Omni antenna should be used in order to sense 360 degree around the node, and for the transmission issue inside the cluster, a directional antenna should be utilized with its direction toward the sink node. After applying on sensing radius it will be known how many nodes are required exactly to be run in the first turn of nodes per network, the second or remaining group of nodes' will be set in timers to run after a while, also the remaining nodes distribution will be in a queue with priority to the very remote nodes, cause they are consuming their energy faster or earlier due to the transmission range is higher.

$N_{\text {required }}=\frac{A}{A_{S}}=$ Required number of nodes per all area

$N_{\text {extra }}=N-N_{\text {req }}=$ Rem.nodes that will be set in timers

The mentioned nodes will be distributed with highest priority to the remotest corona nodes. This issue should be applied and 
tested theoretically for each value of sensing radius $R_{\mathrm{s}}$. If the number of nodes per cluster is too high, then collisions may happen at the sink node when the data is transmitted to the sink node. In this regards, there are four modes can be utilized by each node to transmit their data to the sink.

The proposed model will depend on the Event-Based Delivery mode, which means that the node will not transmit until it senses some information different or newer than the old one, and if there is no updated info, then no transmission will be occurred to the sink mode also the transmission access to the sink node will be assumed as a TDMA mode [16]. In each corona, the transmission range will be different from other coronas, so the energy consumption illustrated by Eq. (14) will be used. By reformulating Eq. (14), then the consumed energy for the seven nodes can be written as in Eq. (26):

$E_{C}=\underbrace{1.11 * r_{\text {sensing }}^{2} * \mu_{2}}_{\text {Sensing part for seven nodes }}+\underbrace{l * r_{\text {transmission }}^{4} * E_{T r}}_{\text {Transmission part }}$

Finally, we can calculate the energy consumed for all the nodes $\mathrm{E}_{\mathrm{C}_{\text {Total }}(\mathrm{j})}$ as follows:

$E_{C_{\text {Total }}}(j)=\sum_{j=1}^{N r} E_{C_{j}}, \ldots, N r=$ number of active nodes

And the objective function equation will be:

$O F(j)=\operatorname{Min}\left(E_{C_{\text {Total }}}(j)\right)$

\section{Power Consumption Optimization \\ 4.1 Power Consumption Optimization using MPSO Algorithm}

The objective of the proposed system is to minimize the energy consumption value illustrated in Eq. (27), so it is required to find the minimum value of the function $E_{C_{\text {Total }}}(j)$ over the interval values of sensing radius. It is assumed here that the sensing radius space is described by, $R_{S_{\text {Min }}} \leq R_{S} \leq$ $R_{S_{\text {Max }}}$ where $\mathrm{R}_{\mathrm{s}}$ is $\mathrm{r}_{\text {Sensing }}$ which is the sensing radius in Eq. (26). We can start to solve this equation by determining the number of particles $P$, which will represent the number of birds in the flock that are going to search for the solution. By picking $P$ (Personal Best $P_{\text {best }}$ ) values for $R_{s}$ over its interval then:

$R s=R s 1, R s 2, R s 3, \ldots \ldots \ldots, R s p$

And by substituting these values in Eq. (30), we can determine the number of solutions, then one of these solutions is local minima between all the solutions, so the value of $R s$ that gives the minimum energy value is called the global best $\left(G_{\text {best }}\right)$.

$E_{C}(i, k)=\underbrace{1.11 * r_{\text {sensing }}^{2}(k) * \mu_{2}}_{\text {Sensing part for seven nodes }}+\underbrace{l * r_{\text {transmission }}^{4} * E_{T r}}_{\text {Transmission part }}$

Where $i$ represent the number of iterations; its values from $i$ till $i_{\max }$ which is the maximum number of iterations required. The parameter $k$ represents the number of particles and $k=1$, $2,3,4, \ldots P$. So another $P$ set of values for $R s$ over the same interval should be chosen to cover all the values in the interval, then the new values will be chosen according to an equation described below:

$$
\begin{aligned}
& V_{\text {new }}(k)=w * V_{\text {old }}(k)+c_{1} * r_{1} *\left(P_{\text {best }}(k)-X_{k}\right)+c_{2} * r_{2} \\
& *\left(G_{\text {best }}-X_{k}\right) \\
& X_{\text {new }}(k)=X_{\text {old }}(k)+V_{\text {new }}(k)
\end{aligned}
$$

Where $V_{\text {old }}, V_{\text {new }}$ are the velocities of the previous and current iteration, $w$ is the inertial weight, $c_{1}, c_{2}$ are the acceleration factors, $X_{\text {old }}$ and $X_{\text {new }}$ are the previous and current values of the selected $R_{s}$.

\subsection{Power Consumption Optimization using ACO Algorithm}

As mentioned before, our aim is to find the minimum value of the function $E_{C_{\text {Total }}}(j)$ over the interval values of sensing radius. So starting to solve this equation by ACO through determining the number of ants $\mathrm{N}$, that will represent the number of ants in the colony that are going to search for the solution or food. Also by assuming that there are a number of permissible discrete values of the design variables $n$. It is required to pick $n$ values of $R s$ over its interval starting from the $R_{s \text { min }}$ and ending with $R_{s_{-} \max }$ and each ant can choose any path from the $\mathrm{n}$ paths from the home to the destination. Assuming an equal amount of pheromone for each path $\left(\tau_{1 j}\right)$, where 1 here means the first iteration and $\mathrm{j}$ refers to the path number so:

$\tau_{1 j}=1, \quad J=1,2,3 \ldots \ldots n$.

For any ant k, the probability of selecting a path (or discrete variable) $\mathrm{x} 1 \mathrm{j}$ is given by:

$P_{i j}=\frac{\tau_{i j}^{(l)}}{\sum_{j=1}^{n} \tau_{i j}^{(l)}} ; i=1,2, \ldots, ; j=1,2,3 \ldots \ldots, n ;$

In order to choose any path, it is required to pick random numbers, then by using (the roulette-wheel selection process) and also by using the cumulative probability range in which the value of the selected $\mathrm{N}$ random falls, the discrete value assumed (or the path selected) by different ants can be seen on the roulette-wheel. The following equations utilized to find out the best and worst paths among the $\mathrm{n}$ paths chosen by different ants:-

$f_{\text {best }}=\left\{f_{k}\right\}_{k=1,2, \ldots \ldots, N}^{\min }$

$f_{\text {worst }}=\left\{f_{k}\right\}_{k=1,2, \ldots \ldots, N}^{\max }$

Assuming that the ants will return to home region and start again in search for food, then by setting the iteration number as $l=2$ and test for the convergence of the process. The process is assumed to have converged if all $N$ ants take the same best path. If convergence is not achieved, assume that all the ants return home and start again in search of food, set the new iteration number as $l=l+1$, and update the pheromones on different arcs (or discrete values of design variables) as:

$\tau_{i j}^{(l)}=\tau_{i j}^{(o l d)}+\sum_{k} \Delta \tau_{i j}^{(k)}$

Where $\tau_{i j}^{(o l d)}$ denotes the pheromone amount of the previous iteration left after evaporation, which is taken as:

$\tau_{i j}^{(o l d)}=(1-\rho) * \tau_{i j}^{(l-1)}$ 
$\sum_{k} \Delta \tau_{i j}^{(k)}$ is the pheromone deposited by the best ant $k$ and the summation extends over all the best ants $\mathrm{k}$ (if multiple ants take the best path). Thus the value of $\sum_{k} \Delta \tau_{i j}^{(k)}$ can be calculated as below:

$\sum_{k} \Delta \tau_{i j}^{(k)}=\frac{\delta * F_{\text {best }}}{F_{\text {worst }}}$

Where $\rho$ the pheromone decay factor and $\delta$ is the scaling parameter. Then for the best path the $\sum_{\mathrm{k}} \Delta \tau_{\mathrm{ij}}^{(\mathrm{k})}$ value will be added to the old pheromone amount, and for the others will be calculated as in Eq. (38). Then repeating the same procedure and here the pheromone of the best option or path will be higher than the others so the space of it in the roulette-wheel will be larger; then the choice of the solution will be directed to the best option until getting the good answer or path by the end of the iterations or stopping criteria.

\section{Simulation Results}

In order to optimize the energy-constrained WSN model, the common parameters illustrated in Table 1 are considered, which are applied in each simulation round.

The proposed system has been implemented using MATLAB 7.6.0 (R2008a) software environment. The computer simulation results show that the presented approach for power consumption minimization is faster than the previous works represented by A-3 and B-3 by 10 times, and the network lifetime is increased by at least 8 times as depicted in Fig. 3 and Fig. 4. Table 2 shows the important results that were obtained in different simulation cases using our model and compared with the previous works under same parameters.

From Table 2, it can be observed that the MPSO algorithm offers superior results in terms of accuracy (99.36\%) in comparison with ACO algorithm (97.92\%). In this regards, MPSO algorithm acts with much better efficiency as computational time minimizes, simple, has stable convergence characteristics, and designed with adaptable inertial weight and acceleration factors than ACO algorithm.

\section{Conclusions}

In this paper, a new model for lifespan prolonging problem of WSN is developed. The proposed system is optimized based on two important nature-inspired algorithms, which are MPSO and ACO to find out the optimum energy consumption for WSN. The comparison of MPSO and ACO shows that MPSO faster than ACO to extract the best results cause the update of the parameters in MPSO is faster and can test the entire solution interval in less time. Additionally, PSO is more accurate than ACO cause in PSO the update of the parameters can cover all the values in the solution interval and always directed to the correct value and solution. Finally, the proposed hexagonal shape model gave good results and approximately similar to that extracted by the triangular and quadrilateral shape models, but the hexagonal is easier to design than the other two previous models because all the seven nodes that will be selected to make a hexagonal network model have the same specifications like sensing radius, coverage area and transmission radius while in the two previous models, it is necessary to select a node in the middle of the network with special dimensions.

Table 1: The simulation parameters.

\begin{tabular}{|c|c|c|c|c|c|c|c|c|c|c|}
\hline \multirow[b]{2}{*}{ 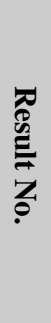 } & \multirow[b]{2}{*}{$\stackrel{7}{3}$} & \multirow{2}{*}{ 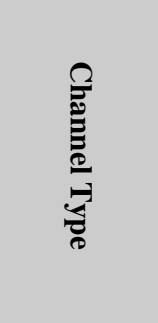 } & \multirow{2}{*}{ 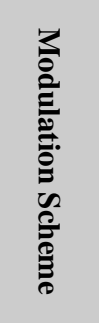 } & \multirow{2}{*}{ 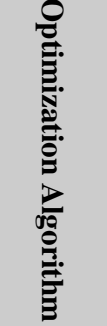 } & \multicolumn{4}{|c|}{ Parameters } & \multicolumn{2}{|l|}{ Results } \\
\hline & & & & & 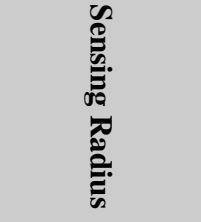 & 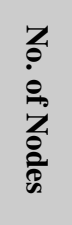 & 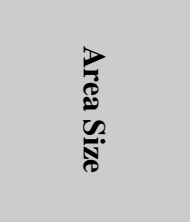 & 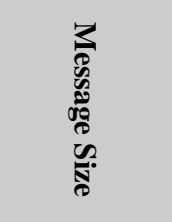 & $\begin{array}{c}\text { First Parameter vs. S } \\
\text { Parameter }\end{array}$ & ond \\
\hline 1 & A & Flat Fading & QPSK & MPSO & $\begin{array}{c}\text { Rs_min }=10 \mathrm{~m} \\
\& \\
\text { Rs_Max }=60 \mathrm{~m}\end{array}$ & 676 & $500 \mathrm{mX} 500 \mathrm{~m}$ & $\begin{array}{c}2000 \\
\text { Bit/Message }\end{array}$ & Lifetime & Rounds \\
\hline 1 & B & Flat Fading & QPSK & MPSO & $\begin{array}{c}\text { Rs_min }=10 \mathrm{~m} \\
\& \\
\text { Rs_Max }=60 \mathrm{~m}\end{array}$ & 676 & $\begin{array}{c}1000 \mathrm{mX} 1000 \\
\mathrm{~m}\end{array}$ & $\begin{array}{c}2000 \\
\text { Bit/Message }\end{array}$ & Lifetime & Rounds \\
\hline 2 & A & Flat Fading & QPSK & MPSO & $\mathrm{Rs}=20 \mathrm{~m}$ & 746 & $\begin{array}{c}5 \text { by } \\
(80 \mathrm{mX} 80 \mathrm{~m})\end{array}$ & & Live Nodes & Rounds \\
\hline 2 & B & Flat Fading & QPSK & $\mathrm{ACO}$ & $\mathrm{Rs}=20 \mathrm{~m}$ & 746 & $\begin{array}{c}5 \text { by } \\
(80 \mathrm{mX} 80 \mathrm{~m})\end{array}$ & & Live Nodes & Rounds \\
\hline 3 & A & Flat Fading & QPSK & MPSO & $\begin{array}{c}\text { Rs_min }=10 \mathrm{~m} \\
\& \\
\text { Rs_Max }=60 \mathrm{~m}\end{array}$ & 500 & $\begin{array}{c}1000 \mathrm{mX} 1000 \\
\mathrm{~m}\end{array}$ & $\begin{array}{c}2000 \\
\text { Bit/Message }\end{array}$ & $\begin{array}{l}\text { Energy consumption Line } \\
\text { in Joules }\end{array}$ & $\begin{array}{l}\text { Time in } \\
\text { seconds }\end{array}$ \\
\hline 3 & B & Flat Fading & QPSK & $\mathrm{ACO}$ & $\begin{array}{c}\text { Rs_min }=10 \mathrm{~m} \\
\& \\
\text { Rs_Max }=60 \mathrm{~m}\end{array}$ & 500 & $\begin{array}{c}1000 \mathrm{mX} 1000 \\
\mathrm{~m}\end{array}$ & $\begin{array}{c}2000 \\
\text { Bit/Message }\end{array}$ & $\begin{array}{l}\text { Energy consumption Line } \\
\text { in Joules }\end{array}$ & $\begin{array}{l}\text { Time in } \\
\text { seconds }\end{array}$ \\
\hline 4 & & Flat Fading & QPSK & & $\mathrm{Rs}=20 \mathrm{~m}$ & 100 & $100 \mathrm{mX} 100 \mathrm{~m}$ & $\begin{array}{c}4000 \\
\text { Bit/Message }\end{array}$ & Live Nodes & Rounds \\
\hline 5 & A & Flat Fading & QPSK & MPSO & $\begin{array}{c}\text { Rs_min }=10 \mathrm{~m} \\
\& \\
\text { Rs_Max }=60 \mathrm{~m}\end{array}$ & 100 & $100 \mathrm{mX} 100 \mathrm{~m}$ & $\begin{array}{c}80 \\
\text { Bit/Message }\end{array}$ & $\begin{array}{l}\text { Energy consumption Line } \\
\text { in Joules }\end{array}$ & $\begin{array}{l}\text { Time in } \\
\text { seconds }\end{array}$ \\
\hline 5 & B & Flat Fading & QPSK & $\mathrm{ACO}$ & $\begin{array}{c}\text { Rs_min }=10 \mathrm{~m} \\
\& \\
\text { Rs_Max }=60 \mathrm{~m}\end{array}$ & 100 & $100 \mathrm{mX} 100 \mathrm{~m}$ & $\begin{array}{c}80 \\
\text { Bit/Message }\end{array}$ & $\begin{array}{l}\text { Energy consumption Line } \\
\text { in Joules }\end{array}$ & $\begin{array}{l}\text { Time in } \\
\text { seconds }\end{array}$ \\
\hline
\end{tabular}




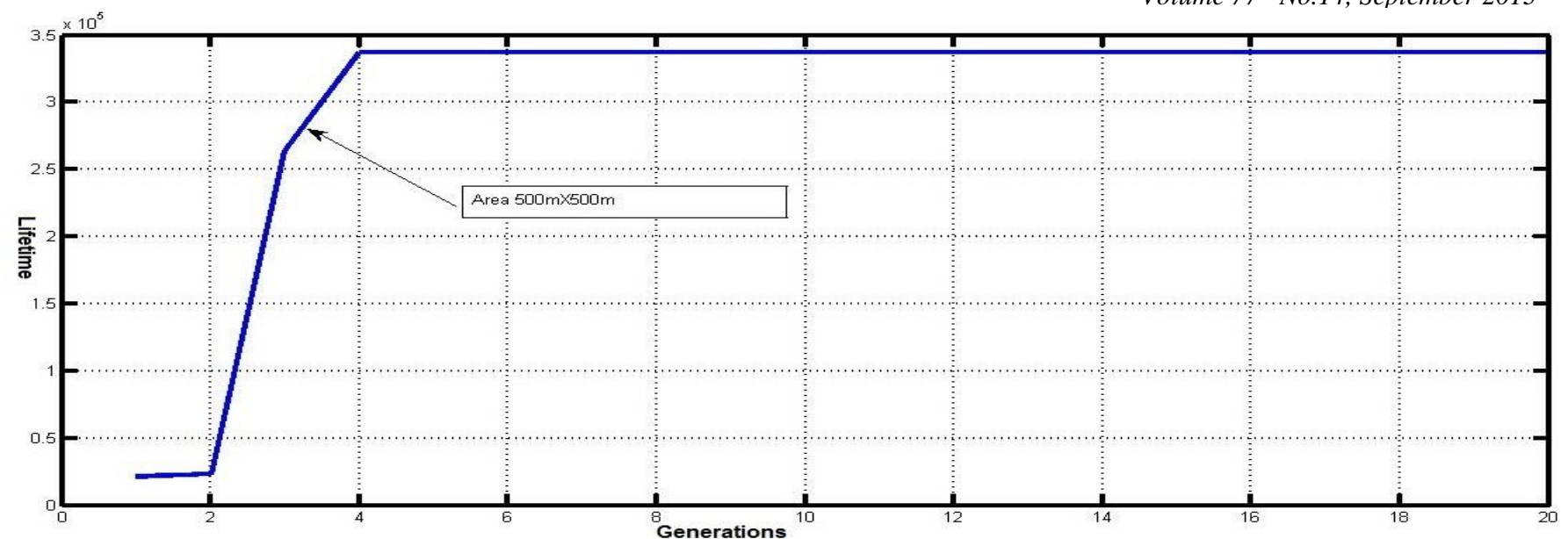

Figure 3: MPSO applied with $500 \times 500 \mathrm{~m} 2$ and 676 nodes.

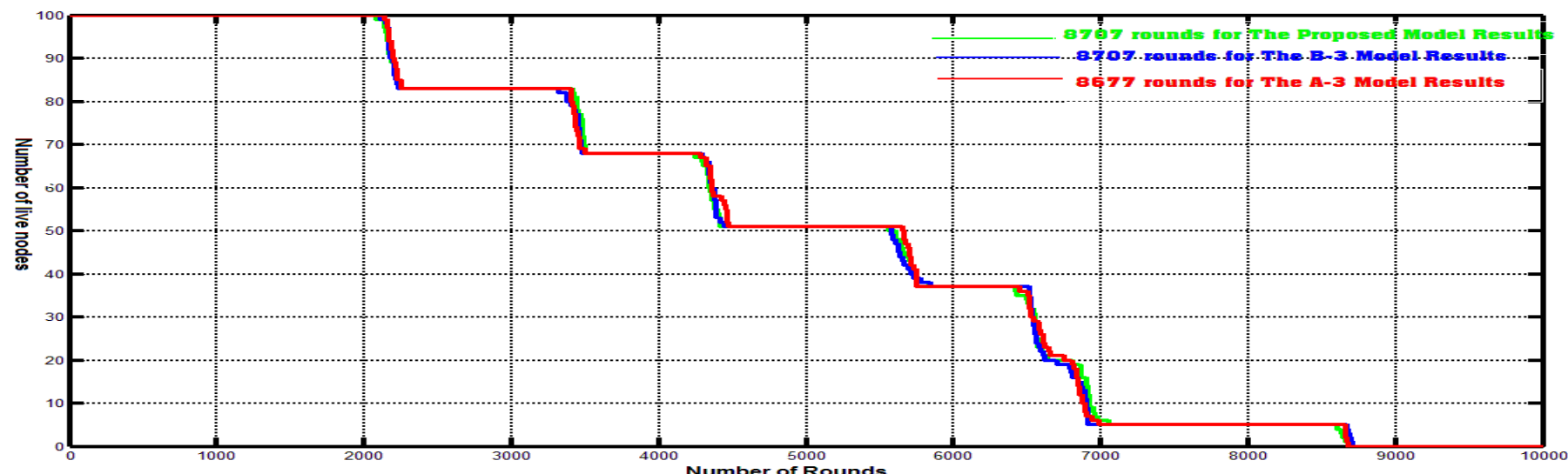

Figure 4a: The application of MPSO algorithm on the three models together.

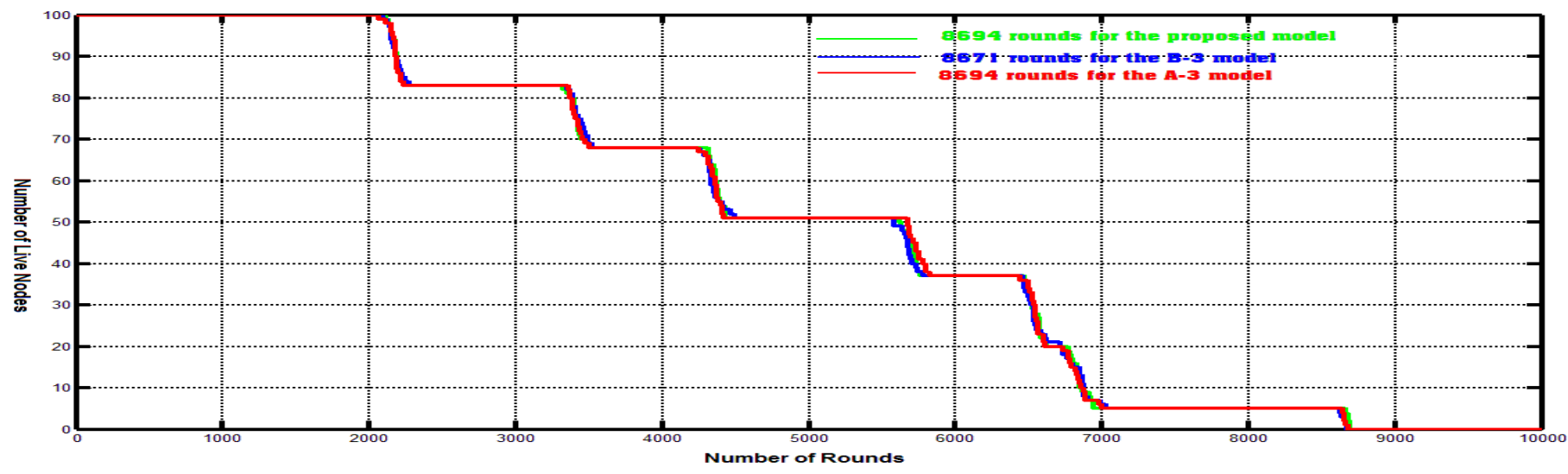

Figure 4b: The application of ACO algorithm on the three models together.

Figure 4: Application of the Proposed Model with A-3 \& B-3 models, 746 Nodes, Rs=20m, Area is 5 grids of $(80 \mathrm{mX80m})$.

Table 2: The collected results based on different simulation parameters.

\begin{tabular}{|c|c|c|c|c|c|c|c|c|c|c|c|c|}
\hline \multirow{2}{*}{ 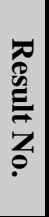 } & \multirow{2}{*}{ 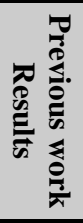 } & \multicolumn{3}{|c|}{$\mathscr{0}$} & \multicolumn{3}{|c|}{8} & \multirow{2}{*}{\multicolumn{2}{|c|}{ Comparison }} & \multirow[b]{2}{*}{ 豆 } & \multicolumn{2}{|c|}{$\begin{array}{c}\text { Enhancement Ratio of } \\
\text { the proposed model }\end{array}$} \\
\hline & & 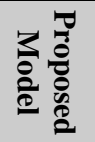 & 虫 & 承 & 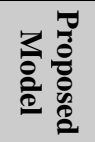 & 岕 & 茪 & & & & है & है \\
\hline 1 & 50 & 5 & N/A & N/A & N/A & N/A & N/A & How fast to $g$ & optimal solution & Generations & $500 \%$ & \\
\hline 2 & 1200 & 8707 & 8877 & 8707 & 8691 & 8694 & 8671 & $\begin{array}{c}\text { Number of live } \\
\text { nodes }\end{array}$ & Number of rounds & Rounds & $725.58 \%$ & $724.25 \%$ \\
\hline 3 & 400 & 22660 & 22668 & 22182 & 22602 & 22621 & 22136 & Energy & Time & Seconds & $5665.00 \%$ & $\begin{array}{c}5650.50 \\
\%\end{array}$ \\
\hline 4 & 1200 & 8717 & 8677 & 8675 & 8717 & 8677 & 8675 & Live nodes & Rounds & Rounds & $726.42 \%$ & $726.42 \%$ \\
\hline 5 & 34 & 336 & 336 & 317 & 336 & 336 & 317 & Energy & Time & Seconds & $988.24 \%$ & $988.24 \%$ \\
\hline
\end{tabular}




\section{REFERENCES}

[1] C. Song, M. Liu, J. Cao, Y. Zheng, H. Gong, and G. Chen, "Maximizing Network Lifetime based on Transmission Range Adjustment in Wireless Sensor Networks," Computer Communications, vol. 32, , no. 11, , pp. 1316-1325, 2009.

[2] L. Bai, L. Zhao, and Z. Liao, "Energy-balanced Parameter-adaptable Protocol Design in Cooperative Wireless Sensor Networks," International Journal of Multimedia and Ubiquitous Engineering, vol. 4, no. 1, , pp. 39-58, 2009.

[3] F. Bagci, T. Ungerer and N.Bagherzadeh, "ESTR Energy Saving Token Ring Protocol for Wireless Sensor Networks," In Proceedings of the 2008 International Conference on Wireless Networks (ICWN), Las Vegas, Nevada, USA, pages 3-9,July 14-17, 2008.

[4] S. Anandamurugan and C. Venkatesh, "Power Saving Scheme (PSS) in Clusters of Heterogeneous Wireless Sensor Networks," International Journal on Computer Science and Engineering (IJCSE), vol. 2, no. 6, pp. 1966-1972, 2010.

[5] C. Srimathi, J. Vaideswaran and S.P. Kumar, "EARQ: Energy Aware Routing for Real-Time Sensor Networks," International Journal of Engineering Science and Technology (IJEST), vol. 3, no. 1, pp. 471-478, 2011.

[6] J. K. Murthy, S. Kumar and A. Srinivas, " Energy Efficient Scheduling in Cross Layer Optimized Clustered Wireless Sensor Networks," International Journal of Computer Science and Communication, vol. 3, no. 1, pp. 149-153, 2012.

[7] S. G. S. P. Yadav and Dr. A. Chitra, "Wireless Sensor Networks - Architectures, Protocols, Simulators and Applications: a Survey," International Journal of Electronics and Computer Science Engineering, vol. 1, No. 4, pp. 1941-1953, 2012.

[8] A. Khosravi, A. Lari, and J. Addeh, " A New Hybrid of Evolutionary and Conventional Optimization Algorithms," Applied Mathematical Sciences, vol. 6, no. 17, pp. $815-825,2012$.
[9] J. Kennedy and R. Eberhart, "Particle Swarm Optimization," Proceedings of IEEE International Conference on Neural Networks, Perth, Australia, vol. IV, pp. 1942-1948, 27 Nov.-1Dec., 1995.

[10] E. Elbeltagi, T. Hegazy, and D. Grierson, “ Comparison among Five Evolutionary-based Optimization Algorithms, " Advanced Engineering Informatics, vol. 19, no. 1, pp.43-53, 2005.

[11] U. OZKAYA, and F. GUNES, “A Modified Particle Swarm Optimization Algorithm and its Application to the Multi-objective FET Modeling Problem," Turkish Journal of Electrical Engineering \& Computer Sciences, vol.20, no.2, pp. 1102-1032, 2012.

[12] M. Chica, O. Cordón, S. Damas, and J. Bautista, “ Including Different Kinds of Preferences in a MultiObjective Ant Algorithm for Time and Space Assembly Line Balancing on Different Nissan Scenarios," Expert Systems with Applications, vol. 38, no. 1, pp. 709-720, 2011.

[13] Z. C. Hlaing, and M. A. Khine, " An Ant Colony Optimization Algorithm for Solving Traveling Salesman

Problem," In Proceedings of International Conference on Information Communication and Management (IPCSIT), Singapore, vol.16, pp. 54-59, 14-16 Oct., 2011.

[14] V. Zalyubovskiy, A. Erzin , S. Astrakov , and H Choo, "Energy-efficient Area Coverage by Sensors with Adjustable ranges," Sensors, vol. 9, no. 4, pp. 24462460, 2009.

[15] J. Wu, and S, Yang, "Coverage Issue in Sensor Networks with Adjustable Ranges," In Proceedings of International Conference on Parallel Processing Workshops(ICPPW04), 14-18 Aug., 2004.

[16] K. Sohraby, D. Minoli, and T. Znati, "Wireless Sensor Networks Technology, Protocols, and Applications," $1^{\text {st }}$ Edition, Wiley \& Sons, Inc., Hoboken, New Jersey, 2007. 